\title{
Unitary representations of super Lie groups
}

\author{
V. S. Varadarajan \\ University of California,Los Angeles,USA,vsv@math.ucla.edu
}

1. Super manifolds and super Lie groups

2. The category of unitary representations of a super Lie group

3. Super systems of imprimitivity on purely even super homogeneous spaces

4. Representations of super semi direct products

5. Super particles and their multiplet structure

\section{Super manifolds and super Lie groups}

1.1. Introduction. Supersymmetry was discovered by the physicists in the early 1970's. Since then an enormous effort has been devoted to constructing supersymmetric versions of the fundamental theories of elementary particles, their fields, and their interactions. In these and the other lectures in this conference an attempt is being made to give a systematic account of the mathematical aspects of supersymmetry.

Everyone knows that the idea of symmetry and the role of group theory in describing it goes back to very ancient times. However it was only in the twentieth century that it became possible to erect a fully satisfactory mathematical theory, namely the theory of Lie groups and their representations, that was adequate and powerful for all the applications. This theory, based on differential and algebraic geometry, was one of the major achievements of mathematics in the last century. But, in the 1970's, 
the physicists, driven by the desire to explain the main features of the physical world at the most fundamental level, came up with a rather remarkable generalization of symmetry, in particular spacetime symmetry, that went beyond conventional limits. Indeed, the idea that one can generalize the notion of symmetry of spacetime beyond what was known for centuries is extraordinary, and one has to thank the physicists for their daring and imagination to have come up with such a generalization. The effort to build a proper mathematical foundation for the discoveries of the physicists led to the development of an entirely new part of mathematics, namely the theory of super manifolds and super Lie groups. This is a development of geometry beyond its conventional limits and it is not clear at this time in what directions it will continue in the future. Certainly the happenings in the world of elementary particles and their fields will have a lot to say about the future growth of what may be called super geometry.

From the historical point of view it is remarkable that already in 1854, Riemann, in his Göttingen inaugural address, had speculated on the structure of space in its microscopic parts, and raised the possibility that the manifold structure may not be present in the infinitely small parts. He also advocated the point of view that we have to look for physics to tell us what this micro-structre should be. Here is what he wrote:

Now it seems that the empirical notions on which the metric determinations of Space are based, the concept of a solid body and a light ray, lose their validity in the infinitely small; it is therefore quite definitely conceivable that the metric relations of Space in the infinitely small do not conform to the hypotheses of geometry; and in fact, one ought to assume this as soon as it permits a simpler way of explaining phenomena.

........An answer to these questions can be found only by starting from that conception of phenomena which has hitherto been approved by experience, for which Newton laid the foundation, and gradually modifying it under the compulsion of facts which cannot be explained by it. Investigations like the one just made, which begin from general concepts, can serve only to ensure that this work is not hindered by too restricted concepts, and that the progress in comprehending the connection of things is not obstructed by traditional prejudices.

The beginnings of supersymmetry can be traced to quantum field theory in which the Hilbert space of one particle states is an orthogonal 
direct sum $\mathcal{H}=\mathcal{H}_{0} \oplus \mathcal{H}_{1}$; here the vectors of $\mathcal{H}_{0}$ (resp. $\mathcal{H}_{1}$ ) define the states where the particle is a Boson (resp. Fermion). Supersymmetries then became transformations that exchanged the Bosonic and Fermionic states. In mathematical terms one may say that the Hilbert spaces of quantum field theory are $\mathbf{Z}_{2}$-graded. The attempt to have a unified way of treating this dual aspect of fundamental particles soon led to linear algebra over $\mathbf{Z}_{2}$-graded spaces, namely, vector spaces with a distinguished decomposition into odd and even subspaces. However the essential point of departure came when physicists like Salam and others realized that one has to construct a graded generalization of classical geometry itself so that, after quantization, one obtains the graded Hilbert spaces so characteristic of quantum field theory. Now the manifolds in classical geometry are non linear and are studied using local coordinates. The ideas of Salam and others then led to the creation of a generalization of classical geometry in which the usual local coordinates were supplemented by a set of Grassmann coordinates. The heuristic meaning of the Grassman coordinates was that they encoded the fermionic aspects of matter, in particular the Pauli exclusion principle that is at the basis of all properties of matter in the bulk. The automorphisms of such super manifolds were to be viewed as the supersymmetries which on quantization led to the Fermi-Bose symmetries of quantum field theory. Because of the novelty and conceptual difficulty of this type of generalization of classical geometry the notion of supersymmetry was formulated at first only infinitesimally, based on the concept of a super Lie algebra. Indeed, the concept of a super Lie algebra itself was born in the attempts by physicists to formulate this type of symmetry. Once the concept of supersymmetry was formulated, albeit infinitesimally, the goals of the physical side of the theory became clear: to work out electrodynamics, and more generally, Yang-Mills theories, and Einstein gravity, under the umbrella of supersymmetry. The structure of these supersymmetric theories is such that they offer many advantages over conventional theories: softer divergences, reduction of possible Lagrangians, and so on. Although the global (as opposed to infinitesimal) formulation of supersymmetry was implicit in the physical theories, it was not fully revealed because of the difficulties in capturing the notion of super Lie groups (as opposed to the technically much simpler notion of super Lie algebras).

In order to understand properly the global geometric nature of super manifolds and supersymmetry it is necessary to look more closely at the nature of super geometry as a profound generalization of classical differen- 
tial geometry and the age-old problem of the structure of the space we live in. The development of classical geometry which goes back to Euclid in ancient times, started in the modern era with Riemann's discovery of Riemannian geometry, evolving ultimately into the modern theory of smooth manifolds and its algebraic counterpart, namely Grothendieck's theory of schemes. Supersymmetry, through the concepts of a super manifold and a super scheme, continues this evolutionary development in an entirely new direction. It adds to the usual commuting local coordinates an additional set of anti-commuting Grassmann coordinates which cannot be seen numerically but which play a fundamental role in determining the theories that are possible on a spacetime with such a local structure. However, the presence of the Grassmann coordinates, which are nilpotent and invisible, has the consequence that the mathematics of the theory of super manifolds can be developed cleanly only by using the methods and techniques that Grothendieck has created in modern algebraic geometry, especially the theory of schemes. This method, called the functorial method by mathematicians, is in fact extremely transparent, and is the one that mirrors completely all the calculations that the physicists make, so much so that one can have the advantage of working in the informal way that is most characteristic of physics, and yet lose nothing in mathematical rigor.

These lectures are in three parts. In the first part ( $\S 1-3)$, which is foundational, I shall try to make precise the concept of a super manifold and its automorphisms, the supersymmetries, and explain the functorial method based on the functor of points. This leads naturally to the concept of a super Lie group and the associated concept of a super Lie algebra. I shall then discuss the concept of a unitary representation of a super Lie group. In the second part $(\S 4)$ I shall discuss the super semi direct products and their irreducible unitary representations. In particular I shall set up the super version of the Mackey-Wigner machine of little groups. In the last part ( $(5)$ I shall apply this theory to describe the super spacetimes and the super Poincaré groups in all dimensions (Minkowski signature), their unitary irreducible representations, and the applications to the classification of super particles and super multiplets.

1.2. The category of ringed spaces. If $X$ is a classical smooth $\left(C^{\infty}=\right.$ infinitely differentiable) manifold, then for each open set $U \subset X$ we have the R-algebra $C^{\infty}(U)$ of smooth real functions on $U$. The property of smoothness is local and the mathematical concept that captures this is 
that of a sheaf, expressed by saying that the assignment

$$
U \longmapsto C^{\infty}(U)
$$

is a sheaf of functions. The rings $C^{\infty}(U)$ are the local rings. If $X, Y$ are two smooth manifolds, a morphism, i.e., a smooth map, from $X$ to $Y$ is a continuous map $\psi$ of $X$ to $Y$ such that for each open $U \subset Y$, the pull-back map $\psi^{*}$ is a homomorphism of $C^{\infty}(U)$ into $C^{\infty}\left(\psi^{-1}(U)\right)$. In this way we have the category of smooth manifolds and smooth maps. But in order to define supermanifolds where there are Grassmann coordinates locally that are numerically unobservable, the above notion of a sheaf of functions has to be generalized significantly in two steps.

The first step of such a generalization is already present in the notion, due to Grothendieck, of a ringed space. This is obtained by giving up the idea that the local rings consist of numerical functions defined on open sets $U$, but are rather abstract commutative rings, denoted usually by $\mathcal{O}_{X}(U)$. Unlike numerical functions the elements of these abstract local rings cannot be restricted to open subsets, and so we have to build the restriction maps as a part of the data defining a ringed space. Thus, for open sets $U, V$ with $V \subset U$, we have restriction maps

$$
\mathcal{O}_{X}(U) \longrightarrow \mathcal{O}_{X}(V),\left.\quad f \longmapsto f\right|_{V}
$$

with the property that if $W \subset V \subset U$ are open, the restriction from $U$ to $W$ is the same as restricting first to $V$ and then to $W$. The local nature of these "functions" is expressed by the requirement that the assignment

$$
U \longmapsto \mathcal{O}_{X}(U)
$$

is a sheaf of rings. The sheaf axiom, which is the exact encoding of locality, is the following: if $U$ is an open set and $\left(U_{i}\right)$ is an open covering of $U$, and if $f_{i} \in \mathcal{O}_{X}\left(U_{i}\right)$ are given, then the following two statements are equivalent:

(a) there is a unique element $f \in \mathcal{O}_{X}(U)$ such that $\left.f\right|_{U_{i}}=f_{i}$ for all indices $i$

(b) for any two indices $i_{1}, i_{2},\left.f_{i_{1}}\right|_{U_{i_{1}} \cap U_{i_{2}}}=\left.f_{i_{2}}\right|_{U_{i_{1}} \cap U_{i_{2}}}$ (as elements of $\left.\mathcal{O}_{X}\left(U_{1} \cap U_{2}\right)\right)$.

A ringed space is thus a pair $\left(X, \mathcal{O}_{X}=\mathcal{O}\right)$ where $X$ is a topological space and $\mathcal{O}$ is a sheaf of commutative rings on $X$. A morphism of a ringed space $X$ into a ringed space $Y$ is a continuous map $\psi: X \longrightarrow Y$ together with a 
pull-back map $\psi^{*}$ of $\mathcal{O}_{Y}$ into $\mathcal{O}_{X}$ above $\psi$; this means that for each open $U \subset Y, \psi^{*}$ is a homomorphism of $\mathcal{O}_{Y}(U)$ into $\mathcal{O}_{X}\left(\psi^{-1}(U)\right)$. Notice that unlike the case when the rings of the sheaves are rings of functions, the pull-backs $\psi^{*}$ for any open set $U \subset Y$ have to be specified as part of the data defining morphisms, and that these pull-backs have to be compatible with restrictions. One thus obtains the category of ringed spaces and their morphisms.

One may view the open sets in $X$ as a category where the set of morphisms from $U$ to $V$ is empty unless $U \subset V$, in which case it consists of the natural inclusion of $U$ in $V$. Then a presheaf is a contravariant functor from the category of open sets in to the category of rings, and the morphisms of ringed spaces are natural transformations between these functors.

Super manifolds are generalizations of ringed spaces. In order to introduce these we have to make the second step of the generalization mentioned above. In the concept of a ringed space introduced above we have made the assumption that the local rings are commutative. However, to reach the concept of a super manifold or more generally a super geometric object such as a super ringed space, we must give up the commutativity assumption on the local rings and make them more general, at least general enough to include the algebras in the commutative and Grassmann coordinates characteristic of the physicists' supersymmetric description of space, time, and matter. Moreover, in order to encode the fact that the supersymmetries exchange the classical and Grassmann coordinates, we must make sure that the local rings do not contain any more information other than the possibility that local Grassmann coordinates can be introduced. It turns out that the correct condition is that the local rings are super commutative. This means that they are $\mathbf{Z}_{2}$-graded, and commutative in the super sense, that is, instead of the relation $a b=b a$ between arbitrary elements of the ring, we will have $a b= \pm b a$ where the sign is always plus unless both $a$ and $b$ are odd, in which case the minus sign is taken. Thus a super ringed space is a pair $\left(X, \mathcal{O}_{X}\right)$ where $\mathcal{O}_{X}$ is a sheaf of super commutative rings on the topological space $X$. A super manifold is a super ringed space with the property that $X$ is a classical smooth manifold, and that locally the sheaf looks like the sheaf of rings on $\mathbf{R}^{p}$ of the form

$$
U \longmapsto C^{\infty}(U) \otimes \Lambda\left[\xi_{1}, \xi_{2}, \ldots, \xi_{q}\right]=C^{\infty}(U)\left[\xi_{1}, \ldots, \xi_{1}\right]
$$


where the $\xi$ are Grassmann variables satisfying

$$
\xi_{i}^{2}=0, \quad \xi_{i} \xi_{j}+\xi_{j} \xi_{i}=0 \quad(i, j=1,2, \ldots, q)
$$

so that $\Lambda\left[\xi_{1}, \ldots, \xi_{q}\right]$ is the exterior algebra over $\mathbf{R}$ in $q$ indeterminates. The reader who is familiar with quantum field theory will recognize in the requirement of super commutativity for the local rings the idea that to make the transition from a description of classical fields to Fermi fields one should replace the classical commutators by anticommutators.

It is clear that one can formulate the notion of a real analytic or even a complex analytic super manifold by replacing the underlying classical manifold by a real or complex analytic super manifold. The natural end of this line of thought is the concept of a super scheme.

1.3. The category of super vector spaces and super algebras. If $A$ is an abelian group and we have a direct sum decomposition $A=\oplus A_{i \in I}$, we say that $A$ is graded by $I$ if $I$ itself is an abelian group and $A_{i} A_{j} \subset$ $A_{i+j}$ for $i, j \in I$. The $A_{i}$ are sometimes referred to as the homogeneous components of $A$. The most common index group $I$ is $\mathbf{Z}$, the ring of integers. If $V$ is a vector space and $S(V), E(V)$ are the symmetric and exterior algebras of $V$, then $S(V)$ and $E(V)$ are graded in a natural manner by $\mathbf{Z}$. In supergeometry grading by $\mathbf{Z}_{2}=\mathbf{Z} / 2 \mathbf{Z}$ is fundamental; if $A$ is $\mathbf{Z}_{2}$-graded, the elements of $A_{0}$ are called even and those of $A_{1}$ are called odd.

The infinitesimal structure of a super manifold at a point is captured by the properties of the category of super vector spaces, namely the category of $\mathbf{Z}_{2}$-graded vector spaces. This is a category with direct sum $\oplus$, dual ${ }^{*}$, and tensor product $\otimes$, and is a prime example of a tensor category where the objects are abstract and the category has the three operations above with suitable functorial properties that imitate those of the vector space category. In particular we have isomorphisms $c_{U V}$,

$$
c_{U V}: U \otimes V \longrightarrow V \otimes U
$$

which (through various requirements such as the hexagon axiom) capture the essential properties of the isomorphisms in the vector space category. There the isomorphisms are given by

$$
c_{U V}: u \otimes v \longmapsto(-1)^{p(u) p(v)} v \otimes u \quad(u \in U, v \in V) .
$$


Here $p(x)$ is the parity of $x$ and is either 0 or 1 . These structural assumptions explain all the characteristic features of the practical use of super vector spaces: sign rule, definition of super commutativity, super Lie algebras, and so on. Moreover they "hide" all the signs in the various definitions. In what follows we shall discuss a few of these. The reader should note that almost everything makes sense for abstract tensor categories and this more general interpretation gives great insight. Finally the field over which all vector spaces, and more generally, the tensor category, is defined, is assumed to be of characteristic 0; in applications it is either $\mathbf{R}$ or $\mathbf{C}$.

For instance an algebra in the super category is an object $A$ with a map $\mu: A \otimes A \longrightarrow A$. It is commutative (which means super commutative) if $\mu=\mu \circ c_{A A}$. For super vector spaces this means the relation $a b=(-1)^{p(a) p(b)} b a$ between any two elements $a, b$ of $A$, a first example of the sign rule: in any classical formula, whenever two odd elements are interchanged, there should appear a minus sign. The $c_{U V}$ can be generalized to an action of the symmetric group $\mathfrak{S}_{N}$ in $N$ letters on $V \otimes V \otimes \ldots \otimes V$ ( $N$ factors). A Lie algebra in the tensor category is an algebra object $\mathfrak{g}$ with multiplication denoted by $[\cdot, \cdot]$, having the properties:

$$
\begin{gathered}
{[\cdot, \cdot]\left(1+c_{\mathfrak{g g}}\right)=0} \\
{[\cdot,[\cdot, \cdot]]\left(1+\sigma+\sigma^{2}\right)=0}
\end{gathered}
$$

where $\sigma$ is the automorphism of $\mathfrak{g} \otimes \mathfrak{g} \otimes \mathfrak{g}$ induced by the permutation $(123) \longrightarrow(312)$. If we unscramble these definitions we obtain the usual working definition of a super Lie algebra. Historically, super Lie algebras first came up in the work of the physicists when the first supersymmetric infinitesimal transformations were written down. Gol'fand-Likhtman and Volkov-Akulov discovered the minimal SUSY extension of the Poincaré Lie algebra in the early 1970's. Wess-Zumino discovered a little later, in 1974, the first example of a simple super Lie algebra, namely the minimal SUSY extension of the conformal Lie algebra. In $1975 \mathrm{~V}$. Kac formally defined super Lie algebras and carried out the super version of the Cartan-Killing classification of simple Lie algebras over $\mathbf{C}$.

Super Lie algebras. A Lie super algebra or super Lie algebra is a super vector space $\mathfrak{g}$ with a bracket [, ] which is a morphism from $\mathfrak{g} \otimes \mathfrak{g}$ to $\mathfrak{g}$ with the following properties:

(a) $[a, b]=-(-1)^{p(a) p(b)}[b, a]$. 
(b) The (super) Jacobi identity

$$
[a,[b, c]]+(-1)^{p(a)[p(b)+p(c)]}[b,[c, a]]+(-1)^{p(c)[p(a)+p(b)]}[c,[a, b]]=0 .
$$

One can hide the signs above by rewriting these relations as we did previously, using $c_{\mathfrak{g g}}$ and the action of $\mathfrak{S}_{3}$ on $\mathfrak{g} \otimes \mathfrak{g} \otimes \mathfrak{g}$. Thus, (b) shows that the super Jacobi identity has the same form as the ordinary Jacobi identity for ordinary Lie algebras. Thus the super Lie algebra is defined in exactly the same manner in the category of super vector spaces as an ordinary Lie algebra is in the category of ordinary vector spaces. It thus appears as an entirely natural object. One might therefore say that a super Lie algebra is a Lie object in the category of super vector spaces.

There is a second way to comprehend the notion of a super Lie algebra which is more practical. The bracket is skew symmetric if one of the elements is even and symmetric if both are odd. The super Jacobi identity has 8 special cases depending on the parities of the three elements $a, b, c$. If all three are even the definition is simply the statement that $\mathfrak{g}_{0}$ is a (ordinary) Lie algebra. The identities with 2 even and 1 odd say that $\mathfrak{g}_{1}$ is a $\mathfrak{g}_{0}$-module. The identities with 2 odd and 1 even say that the bracket

$$
\mathfrak{g}_{1} \otimes \mathfrak{g}_{1} \longrightarrow \mathfrak{g}_{0}
$$

is a symmetric $\mathfrak{g}_{0}$-map. Finally, the identities for all three odd elements reduce to

$$
[a,[b, c]]+\ldots+\ldots=0 \quad\left(a, b, c \in \mathfrak{g}_{1}\right)
$$

where $+\ldots+\ldots$ is cyclic summation in $a, b, c$. It is not difficult to see that the last requirement is equivalent to

$$
[a,[a, a]]=0 \quad\left(a \in \mathfrak{g}_{1}\right) .
$$

Thus a super Lie algebra is a super vector space $\mathfrak{g}$ on which a bilinear bracket $[$,$] is defined such that$

(a) $\mathfrak{g}_{0}$ is an ordinary Lie algebra for $[$,$] .$

(b) $\mathfrak{g}_{1}$ is a $\mathfrak{g}_{0}$-module for the action $a \longmapsto \operatorname{ad}(a): b \longmapsto[a, b]\left(b \in \mathfrak{g}_{1}\right)$.

(c) $a \otimes b \longmapsto[a, b]$ is a symmetric $\mathfrak{g}_{0}$-module map from $\mathfrak{g}_{1} \otimes \mathfrak{g}_{1}$ to $\mathfrak{g}_{0}$.

(d) For all $a \in \mathfrak{g}_{1}$, we have $[a,[a, a]]=0$.

Except for (d) the other conditions are linear and can be understood within the framework of ordinary Lie algebras and their representations. The 
condition (d) is nonlinear and is the most difficult to verify in applications when Lie super algebras are constructed by putting together an ordinary Lie algebra and a module for it satisfying (a)-(c).

If $A$ is a super algebra, we define

$$
[a, b]=a b-(-1)^{p(a) p(b)} b a \quad(a, b \in A) .
$$

It is then an easy verification that $[$,$] converts A$ into a super Lie algebra. It is denoted by $A_{L}$ but often we omit the suffix $L$. If $A=\operatorname{End}(V)$, we often write $\mathfrak{g l}(V)$ for the corresponding Lie algebra; if $V=\mathbf{R}^{p \mid q}$ we write $\mathfrak{g l}(p \mid q)$ for $\mathfrak{g l}(V)$.

Let $\mathfrak{g}$ be a super Lie algebra and for $X \in \mathfrak{g}$ let us define

$$
\text { ad } X: \mathfrak{g} \longrightarrow \mathfrak{g}, \quad \text { ad } X(Y)=[X, Y]
$$

Then

$$
\operatorname{ad}: X \longmapsto \operatorname{ad} X
$$

is a morphism of $\mathfrak{g}$ into $\mathfrak{g l}(\mathfrak{g})$. The super Jacobi identity is just the relation

$$
[\operatorname{ad} X, \operatorname{ad} Y]=\operatorname{ad}[X, Y] \quad(X, Y \in \mathfrak{g}) .
$$

The development of super linear algebra including the theory of modules over super commutative rings has no real surprises till we come to the generalization of the determinant, the Berezinian. Let $R$ be a super commutative algebra over a field $k$ of characteristic 0 and $R^{p \mid q}$ be the free module of dimension $p \mid q$ over $R$. Let $\operatorname{GL}(p \mid q)(R)$ be the group of invertible even morphisms of $R^{p \mid q}$. Then the Berezinian is a morphism of $\operatorname{GL}(p \mid q)(R)$ into $R_{0}^{\times}$(the group of units of the even part $R_{0}$ of $R$ ) given by

$$
\operatorname{Ber}(x)=\operatorname{det}\left(A-B D^{-1} C\right) \operatorname{det}(D)^{-1}
$$

where

$$
x=\left(\begin{array}{cc}
A & B \\
C & D
\end{array}\right)
$$

We have

$$
\operatorname{Ber}(x y)=\operatorname{Ber}(x) \operatorname{Ber}(y)
$$

This is the superversion of the determinant, discovered by F. A. Berezin, one of the pioneers of super algebra and super analysis. Since the entries 
of $B$ and $C$ are nilpotent, $x$ is invertible if and only if $A$ and $D$, whose entries are in the commutative ring $R_{0}$, are invertible. The usual determinant in the commutative category can be interpreted as the result of an action on the top part of the exterior algebra; for the super category the generalization of such an interpretation is homological and lies at a deeper level.

1.4. Properties of super manifolds. We outline in brief some of the properties of the category of super manifolds.

Functor of points. Exactly as in the theory of schemes there is another notion of points of a super manifold which is the true geometric one. Let $X$ be a super manifold. For any super manifold $T$ we write $X(T)$ for the set of maps $T \longrightarrow X$ and view the elements of $X(T)$ as the $T$-points of $X$. If $T$ is a single point viewed as a purely even manifold, $X(T)$ is the set of topological points of $X . X(T)$ is a contravariant functor in $T$ and for any super manifold $Y$, the set $\operatorname{Hom}(X, Y)$ can be recovered precisely as the set of natural transformations $X(T) \longrightarrow Y(T)$ (Yoneda's lemma). One can use this to define the products of super manifolds: the product $X_{1} \times \ldots \times X_{N}$ is the super manifold whose $T$-points are $X_{1}(T) \times \ldots \times X_{N}(T)$. In this context we introduce the usual definition of when a contravariant functor $T \longmapsto f(T)$ is representable, namely when there is a super manifold $M$ such that $M(T)=f(T)$.

Morphisms. Because the local rings $C^{\infty}\left(x_{1}, \ldots, x_{p}\right)\left[\theta_{1}, \ldots \theta_{q}\right]$ are not algebraically generated by the $x_{1}, \ldots, x_{p}, \theta_{1}, \ldots \theta_{q}$ one has to be apparently careful in defining morphisms. Actually the situation is exactly as in the algebraic case, and this is the reason why the theory of super manifolds can be developed for the most part as the theory of classical manifolds. If $M, N$ is a super manifolds and $x_{1}, \ldots x_{p}, \theta_{1} \ldots \theta_{q}$ are coordinates on $N$, to define a morphism from $M$ to $N$ it is sufficient to specify the images of th $x_{i}, \theta_{j}$.

Differential calculus. Because of the above result on morphisms, one has a theory of differentiation on super manifolds that is completely analogous to the theory on classical manifolds. The differential criteria for a map to be a local diffeomorphism, the structure of submanifolds, etc remain essentially the same.

Integral calculus. Let

$$
\theta^{I}=\theta^{i_{1}} \theta^{i_{2}} \ldots \theta^{i_{k}} \quad I=\left(i_{\mu}\right),\left\{i_{1}<i_{2}<\ldots<i_{k}\right\} .
$$


On

$$
\Lambda=\mathbf{R}\left[\theta^{1}, \ldots, \theta^{q}\right]
$$

the integral is a linear map

$$
a \longmapsto \int a d^{q} \theta
$$

defined by

$$
\int \theta^{I} d^{q} \theta= \begin{cases}0 & \text { if }|I|<q \\ 1 & \text { if } I=: Q=\{1,2, \ldots, q\} .\end{cases}
$$

Integration is also differentiation:

$$
\int=\left(\frac{\partial}{\partial \theta^{q}}\right)\left(\frac{\partial}{\partial \theta^{q-1}}\right) \ldots\left(\frac{\partial}{\partial \theta^{1}}\right) .
$$

In the local ring with coordinates $x^{i}, \theta^{j}$,

$$
\int s d^{p} x d^{q} \theta=\int s_{Q} d^{p} x \quad\left(s=\sum_{I} s_{I} \theta^{I}\right) .
$$

The change of variables formula. For a morphism given locally as

$$
\psi:(x, \theta) \longmapsto(y, \varphi)
$$

we define the Jacobian matrix

$$
J=\left(\begin{array}{cc}
\frac{\partial y}{\partial x} & -\frac{\partial y}{\partial \theta} \\
\frac{\partial \varphi}{\partial x} & \frac{\partial \varphi}{\partial \theta}
\end{array}\right)
$$

Then

$$
\int s=\int \psi^{*}(s) \operatorname{Ber}(J \psi)
$$

for compactly supported sections of the local ring. For arbitrary manifolds we use partitions of unity as in the classical case. This beautiful formula goes back to Berezin. The justification for the peculiar definition of integration in the anticommuting variables is the change of variables formula.

The global section functor. Finally, we have the global section functor which associates to any $C^{\infty}$ manifold $M$ the super algebra $A(M)$ of global sections, namely, $A(M)=\mathcal{O}_{M}(M)$. It is possible to show that 
$M$ can be recovered from $A(M)$, more precisely, the functor of globals sections is fully faithful.

Heuristic conception of a super manifold. In calculations with super manifolds physicists work with coordinates and manipulate the odd variables more or less on the same footing as the classical commuting coordinates. The functor of points approach is essentially a way to make sense of such calculations.

The intuitive picture of $M$ is that of a classical smooth manifold $|M|$ surrounded by a grassmannian cloud. The cloud cannot be seen: in any measurement the odd variables will be 0 because they are nilpotent. Thus measurement sees only the underlying classical manifold $|M|$. Nevertheless the presence of the cloud eventually has consequences that are striking.

1.5. Super Lie groups and their Lie algebras. Super Lie groups are group objects in the category of super manifolds. Thus to say that $G$ is a super Lie group is to say that $G$ is a super manifold, and that we have morphisms

$$
\mu: G \times G \longrightarrow G, \quad \iota: G \longrightarrow G
$$

that have the associativity properties usually ascribed to multiplication and inverse, together with the identity element

$$
e:\{\text { point }\} \longrightarrow G
$$

From the perspective of functor of points the functor $G(T)$ takes values in groups, so that a super Lie group may be defined as a contravariant functor from the category of super manifolds to the category of groups, which is representable. The theory of super Lie groups may be built up in the same way as the theory of classical Lie groups, but some of the technical aspects become more involved because things have to be done using the functor of points.

One can define on any super Lie group the notion of left (or right) invariant vector fields, and hence the notion of a Lie algebra of a super Lie group. This is a super Lie algebra and the correspondence between $G$ and its super Lie algebra $\mathfrak{g}$ has essentially all the features of the classical correspondence.

Super Harish-Chandra pairs. Of great importance for us is a theorem which allows one to describe a super Lie group in more concrete 
terms. Given a super Lie group $G$ we have a pair $\left(G_{0}, \mathfrak{g}\right)$ where $G_{0}$ is the classical Lie group underlying $G$, and $\mathfrak{g}$ is the super Lie algebra of $G$. We have

(1) $\operatorname{Lie}\left(G_{0}\right)=\mathfrak{g}_{0}$.

(2) $G_{0}$ has an action on $\mathfrak{g}$ and its differential is the adjoint action of $\mathfrak{g}_{0}$ on $\mathfrak{g}$.

Such pairs $\left(G_{0}, \mathfrak{g}\right)$ can be also introduced a priori without reference to a super Lie group. They are then called super Harish-Chandra pairs. The basic theorem, which is fundamental for us, is the result that

$$
G \longmapsto\left(G_{0}, \mathfrak{g}\right)
$$

is a covariant functor which is an equivalence of categories from the category of super Lie groups to the category of super Harish-Chandra pairs. 


\section{The category of unitary representations of a super Lie group}

2.1. Introduction. The study of unitary representations of super Lie algebras began not long after super Lie algebras were discovered and classified. However unitary representations of super Lie groups are a different story because of the subtle manner in which a super Lie group differs from an ordinary Lie group, and their study has lagged far behind that of the representations of super Lie algebras. In this section I shall discuss how to make the correct definition for a unitary representation of a super Lie group. It seems likely that further developments of the representation theory of super Lie groups will require a theory of representations of super Lie groups in Banach and even Frechet spaces, but we shall not take up these generalizations here.

Our point of view is that a super Lie group may be identified with a super Harish-Chandra pair $\left(G_{0}, \mathfrak{g}\right)$. This is reasonable, since as we saw earlier, the category of super Lie groups is equivalent to the category of super Harish-Chandra pairs. Let me recall that $\left(G_{0}, \mathfrak{g}\right)$ is a super HarishChandra pair if $G_{0}$ is a classical Lie group and $\mathfrak{g}$ is a super Lie algebra with an action of $G_{0}$ on it such that

(i) $\operatorname{Lie}\left(G_{0}\right)=\mathfrak{g}_{0}$ (=the even part of $\left.\mathfrak{g}\right)$.

(ii) The action of $G_{0}$ on $\mathfrak{g}$ is the adjoint action of $G_{0}$; more precisely, the adjoint action of $\mathfrak{g}_{0}$ on $\mathfrak{g}$ is the differential of the action of $G_{0}$ on $\mathfrak{g}$.

We shall make no distinction between super Lie groups and super HarishChandra pairs. If we carry over this principle to representations we are led to the idea that a representation of a super Lie group $\left(G_{0}, \mathfrak{g}\right)$ may be thought of as a triple $\left(\pi_{0}, \gamma, \mathcal{H}\right)$ where $\pi_{0}$ is an even representation of $G_{0}$ in a super Banach (or Frechet) space $\mathcal{H}$ (this means that $\pi_{0}(g)$ is even for all $g \in G_{0}$ ), and $\gamma$ is a super representation of $\mathfrak{g}$ in $\mathcal{H}$ compatible with $\pi_{0}$. We shall only be interested in the case of unitary representations. This means that $\mathcal{H}$ is a super Hilbert space, $\pi_{0}$ is unitary, and $\gamma$ satisfies an appropriate condition that reflects the unitarity of the representation. It turns out that one should require that $\gamma(X)$ be skew self adjoint in the super sense for all $X \in \mathfrak{g}$. There is in addition a technical point: for $X \in \mathfrak{g}_{1},[X, X] \in \mathfrak{g}_{0}$ and $\gamma([X, X])=2 \gamma(X)^{2}$, and so the operators $\gamma(X)\left(X \in \mathfrak{g}_{1}\right)$ will in general be unbounded; this means that there are 
domain considerations to be taken care of. In the next section we shall address both these points.

2.2. The basic definitions. All sesquilinear forms are linear in the first argument and conjugate linear in the second (opposite to the physicists' convention regrettably!). A super Hilbert space is a $\mathbf{Z}_{2}$-graded Hilbert space $\mathcal{H}$ where the $\mathcal{H}_{i}(i=0,1)$ are closed mutually orthogonal subspaces. If $\langle x, y\rangle$ is defined as $i^{p(x) p(y)}(x, y)$, then $\langle x, y\rangle$ is an even super Hermitian form. For bounded operators $T: \mathcal{H} \longrightarrow \mathcal{H}$ the adjoint with respect to the super form is denoted by $T^{\dagger}$ and is related to the usual Hilbert space adjoint by $T^{\dagger}=T^{*}$ or $-i T^{*}$ according as $T$ is even or odd. We define $T^{\dagger}$ for unbounded $T$ by this rule in terms of $T^{*}$.

In order to motivate our definition of unitary representations of super Lie groups we begin by observing that in the even case of an ordinary Lie group, the unitarity of a representation $\pi$ of it is usually expressed at the infinitesimal level as follows: if $d \pi$ is the representation of the Lie algebra, then for all $X$ in the Lie algebra the operators $d \pi(X)$ are skew Hermitian. If we carry this over to the super setting the condition should be that the $d \pi(X)$ should be skew super Hermitian for all $X \in \mathfrak{g}$. This means that for $X \in \mathfrak{g}_{0}$ we should require that $d \pi(X)$ should be skew Hermitian while for $X \in \mathfrak{g}_{1}$ we should have $d \pi(X)^{*}=-i d \pi(X)$, ignoring domain considerations. Let

$$
\zeta=e^{-i \pi / 4}
$$

Then this last condition can be written as the condition that

$$
\rho(X)=\zeta d \pi(X) \quad\left(X \in \mathfrak{g}_{1}\right)
$$

be Hermitian symmetric. Thus at the formal level we define a unitary representation of $\left(G_{0}, \mathfrak{g}\right)$ to be a triple $\left(\pi_{0}, \gamma, \mathcal{H}\right)$ where $\pi_{0}$ is an even unitary representation in a super Hilbert space $\mathcal{H}, \gamma$ is a representation of $\mathfrak{g}$ that is compatible with the action of $G_{0}$ on $\mathfrak{g}$, with $\gamma=d \pi_{0}$ on $\mathfrak{g}_{0}$ and $\rho(X)=\zeta \gamma(X)$ Hermitian symmetric for all $X \in \mathfrak{g}_{1}$. In other words, unitary representations are triples $\left(\pi_{0}, \rho, \mathcal{H}\right)$ with the appropriate compatibility and symmetry conditions described above.

To make this definition precise we have to bring in the domains of the operators $\rho(X)$ for $X \in \mathfrak{g}_{1}$. It is natural to start with the assumption that they should have a common dense domain. Initially we shall suppose that this domain is the space of all differentiable vectors for $\pi_{0}$, but this will 
be relaxed later and we shall see that even if one starts with a different domain, the compatibility and symmetry conditions force the operators $\rho(X)\left(X \in \mathfrak{g}_{1}\right)$ to be well defined on the space of all differentiable vectors for $\pi_{0}$.

We therefore make the following definition. A unitary representation of a super Lie group $\left(G_{0}, \mathfrak{g}\right)$ is a triple $\left(\pi_{0}, \rho, \mathcal{H}\right)$ with the following properties:

(i) $\pi_{0}$ is an even unitary representation of $G_{0}$ in the super Hilbert space $\mathcal{H}$.

(ii) $\rho$ is a linear map of $\mathfrak{g}_{1}$ into the subspace of odd endomorphisms of $C^{\infty}\left(\pi_{0}\right)$. Here $C^{\infty}\left(\pi_{0}\right)$ is the space of differentiable vectors for $\pi_{0}$ (it is super linear, i.e., it contains the odd and even components of each of its elements).

(iii) $\rho$ satisfies the requirements below:

(a) $\rho\left(g_{0} X\right)=\pi_{0}\left(g_{0}\right) \rho(X) \pi_{0}\left(g_{0}\right)^{-1}\left(X \in \mathfrak{g}_{1}, g_{0} \in G_{0}\right)$ (compatibility of $\rho$ with $\left.\pi_{0}\right)$.

(b) $\rho(X)$ with domain $C^{\infty}\left(\pi_{0}\right)$ is symmetric for all $X \in \mathfrak{g}_{1}$. This means that the adjoint $\rho(X)^{*}$ is an extension of $\rho(X)$ for $X \in \mathfrak{g}_{1}$.

(c) $-i d \pi_{0}([X, Y])=\rho(X) \rho(Y)+\rho(Y) \rho(X)\left(X, Y \in \mathfrak{g}_{1}\right)$ on $C^{\infty}\left(\pi_{0}\right)$.

Theorem 1. If $\left(\pi_{0}, \rho, \mathcal{H}\right)$ is a unitary representation of $\left(G_{0}, \mathfrak{g}\right)$, then for any $X \in \mathfrak{g}_{1}$ the operator $\rho(X)$ with domain $C^{\infty}\left(\pi_{0}\right)$ is essentially self adjoint, and

$$
\pi: X_{0}+X_{1} \longmapsto d \pi_{0}\left(X_{0}\right)+\zeta^{-1} \rho\left(X_{1}\right)\left(X_{0} \in \mathfrak{g}_{0}, X_{1} \in \mathfrak{g}_{1}\right)
$$

is a super representation of the super Lie algebra $\mathfrak{g}$ in $C^{\infty}\left(\pi_{0}\right)$ compatible with $\pi_{0}$.

The choice of $C^{\infty}\left(\pi_{0}\right)$ as the domain for the operators $\rho(X)$, while natural, might seem arbitrary. For instance one might use the analytic vectors instead of the differentiable ones. It turns out that this objection is illusory, i.e., any alternative system leads always to a unitary representation in the above sense. This makes it clear that in spite of appearances our definition of a unitary representation of a super Lie group is a very viable notion. To make these remarks more precise let us consider a system $\left(\pi_{0}, \rho, \mathcal{B}, \mathcal{H}\right)$ with the following properties.

(i) $\mathcal{B}$ is a dense super linear subspace of $\mathcal{H}$ invariant under $\pi_{0}, \pi_{0}$ being an even unitary representation of $G_{0}$ in the super Hilbert space $\mathcal{H}$. 
Moreover $\mathcal{B} \subset D\left(d \pi_{0}(Z)\right)$ for all $Z \in\left[\mathfrak{g}_{1}, \mathfrak{g}_{1}\right], D(A)$ denoting the domain of the operator $A$. Here for any $Z \in \mathfrak{g}_{0}$, we write $-i d \pi_{0}(Z)$ for the unique self adjoint operator in $\mathcal{H}$ such that $\pi_{0}(\exp t Z)=$ $\left.\exp t d \pi_{0}(Z)\right)$ for all $t \in \mathbf{R}$.

(ii) Each $\rho(X)$ for $X \in \mathfrak{g}_{1}$ is a linear operator in $\mathcal{H}$ with $\mathcal{B} \subset D(\rho(X))$ such that $X \longmapsto \rho(X)$ is a linear map of $\mathfrak{g}_{1} \operatorname{into} \operatorname{Hom}(\mathcal{B}, \mathcal{H})$. Moreover,

(a) $\rho(X)$ is symmetric for all $X \in \mathfrak{g}_{1}$.

(c) $\rho(X) \mathcal{B}_{i} \subset \mathcal{H}_{i+1(\bmod 2)}$ for all $X \in \mathfrak{g}_{1}$.

(d) $\rho$ is compatible with $\pi_{0}$, i.e.,

$$
\pi_{0}(g) \rho(X) \pi_{0}(g)^{-1}=\rho(g X)
$$

on $\mathcal{B}$ for $g \in G_{0}, X \in \mathfrak{g}_{1}$.

(e) $\rho(X) \mathcal{B} \subset D(\rho(Y))$ for all $X, Y \in \mathfrak{g}_{1}$ and

$$
-i d \pi_{0}([X, Y])=\rho(X) \rho(Y)+\rho(Y) \rho(X)
$$

for all $X, Y \in \mathfrak{g}_{1}$ on $\mathcal{B}$.

Theorem 2. Let $\left(\pi_{0}, \rho, \mathcal{B}, \mathcal{H}\right)$ be as above. Then for any $X \in \mathfrak{g}_{1}, \rho(X)$ is essentially self adjoint on $\mathcal{B}$ and $C^{\infty}\left(\pi_{0}\right) \subset D(\overline{\rho(X)})$. Let us write $\bar{\rho}(X)$ for the restriction of $\overline{\rho(X)}$ to $C^{\infty}\left(\pi_{0}\right)$. Then $\left(\pi_{0}, \bar{\rho}, \mathcal{H}\right)$ is a unitary representation of $\left(G_{0}, \mathfrak{g}\right)$. If $\left(\pi_{0}, \rho^{\prime}, \mathcal{H}\right)$ is a unitary representation of $\left(G_{0}, \mathfrak{g}\right)$ such that $\mathcal{B} \subset D\left(\overline{\rho^{\prime}(X)}\right)$ and $\overline{\rho^{\prime}(X)}$ restricts to $\rho(X)$ on $\mathcal{B}$ for all $X \in \mathfrak{g}_{1}$, then $\rho^{\prime}=\bar{\rho}$.

Remark. For operators $A, B$ in $\mathcal{H}$ write $A \prec B$ if $D(A) \subset D(B)$ and $B$ coincides with $A$ on $D(A)$. Let $A$ be a densely defined symmetric operator which is essentially self adjoint. Then $\bar{A}$, the closure of $A$ (which exists because $A$ is symmetric), is the unique self adjoint extension of $A$; moreover, if $B$ is any symmetric operator such that $A \prec B$, we have $\bar{A}=\bar{B}$ so that $A \prec B \prec \bar{A}=\bar{B}$. Thus, as a consequence of the first statement in Theorem 2 we see that extension by closure of $\rho(X)$ is self adjoint and its domain contains $C^{\infty}\left(\pi_{0}\right)$. This is the key fact in the theorems.

In the classical theory of representations of semi simple or reductive Lie groups the $K$-finite vectors play a decisive role. The space of $K$-finite vectors, although in general a subspace of the space of analytic vectors, is not invariant under the group but only under the Lie algebra. So it would 
appear useful to have a variant of Theorem 2 where $\mathcal{B}$ is a subspace of $C^{\omega}\left(\pi_{0}\right)$, the space of analytic vectors of $\pi_{0}$, with $\mathcal{B}$ stable under $\mathfrak{g}$ rather than $G_{0}$. We then have the following theorem.

Theorem 3. $(i)$ If $\left(\pi_{0}, \rho, \mathcal{H}\right)$ is a unitary representation of $\left(G_{0}, \mathfrak{g}\right)$, then the $\rho(X)$ for $X \in \mathfrak{g}_{1}$ map $C^{\omega}\left(\pi_{0}\right)$ into itself, and $\pi$ as in Theorem 1is a representation of $\mathfrak{g}$ in $C^{\omega}\left(\pi_{0}\right)$. (ii) Let $G_{0}$ be connected, let $\mathcal{B}$ be a dense super linear subspace of $C^{\omega}\left(\pi_{0}\right)$, and let $\pi$ be a representation of $\mathfrak{g}$ in $\mathcal{B}$ such that $\pi(Z) \prec d \pi_{0}(Z)$ for all $Z \in \mathfrak{g}_{0}$ and $\rho(X)=\zeta \pi(X)$ symmetric for $X \in \mathfrak{g}_{1}$. Then for any $X \in \mathfrak{g}_{1}, \rho(X)$ is essentially self adjoint on $\mathcal{B}$ and $C^{\infty}\left(\pi_{0}\right) \subset D(\overline{\rho(X)})$. Let us write $\bar{\rho}(X)$ for the restriction of $\overline{\rho(X)}$ to $C^{\infty}\left(\pi_{0}\right)$. Then $\left(\pi_{0}, \bar{\rho}, \mathcal{H}\right)$ is a unitary representation of $\left(G_{0}, \mathfrak{g}\right)$. Finally, if $\left(\pi_{0}, \rho^{\prime}, \mathcal{H}\right)$ is a unitary representation of $\left(G_{0}, \mathfrak{g}\right)$ such that $\mathcal{B} \subset D\left(\overline{\rho^{\prime}(X)}\right)$ and $\rho^{\prime}(X)$ restricts to $\rho(X)$ on $\mathcal{B}$ for all $X \in \mathfrak{g}_{1}$, then $\rho^{\prime}=\bar{\rho}$.

With these theorems we have realized our objective, namely to introduce a viable category of unitary representations of a super Lie group. A morphism from $\Pi=\left(\pi_{0}, \rho, \mathcal{H}\right)$ to $\Pi^{\prime}=\left(\pi_{0}^{\prime}, \rho^{\prime}, \mathcal{H}^{\prime}\right)$ is a bounded linear map $A\left(\mathcal{H} \longrightarrow \mathcal{H}^{\prime}\right)$ that intertwines $\pi_{0}, \pi_{0}^{\prime}$ and $\rho, \rho^{\prime}$. Note that as soon as $A$ intertwines $\pi_{0}$ and $\pi_{0}^{\prime}$, it maps $C^{\infty}\left(\pi_{0}\right)$ into $C^{\infty}\left(\pi_{0}^{\prime}\right)$, and so the statement that $A$ intertwine $\rho, \rho^{\prime}$ makes sense. If $A$ an isomorphism and unitary we speak of unitary equivalence. $\Pi^{\prime}$ is a subrepresentation of $\Pi$ if $\mathcal{H}^{\prime}$ is a closed graded subspace of $\mathcal{H}$ invariant under $\pi_{0}, \mathcal{H}^{\prime} \cap C^{\infty}\left(\pi_{0}\right)$ is invariant under $\rho$, and $\pi_{0}^{\prime}$ (resp. $\rho^{\prime}$ ) is the restriction of $\pi_{0}$ (resp. $\rho$ ) to $\mathcal{H}^{\prime}$ (resp. $\mathcal{H} \cap C^{\infty}\left(\pi_{0}\right)$ ). If $\Pi^{\prime}$ is a proper nonzero subrepresentation of $\Pi, \mathcal{H}^{\prime \prime}=\mathcal{H}^{\prime \perp}$, and $\pi_{0}^{\prime \prime}$ is the restriction of $\pi_{0}$ to $\mathcal{H}^{\prime \prime}$, then $C^{\infty}\left(\pi_{0}\right)$ is the direct sum od $C^{\infty}\left(\pi_{0}^{\prime}\right)$ and $C^{\infty}\left(\pi_{0}^{\prime \prime}\right)$, and it follows from the essential self adjointness of $\rho(X)\left(X \in \mathfrak{g}_{1}\right)$ that $C^{\infty}\left(\pi^{\prime \prime}\right)$ is stable under all the $\rho(X)$; if $\rho^{\prime \prime}$ is the restriction of $\rho$ to $C^{\infty}\left(\pi_{0}^{\prime \prime}\right)$, then $\Pi^{\prime \prime}:=\left(\pi_{0}^{\prime \prime}, \rho^{\prime \prime}, \mathcal{H}^{\prime \prime}\right)$ is a unitary representation of $\left(G_{0}, \mathfrak{g}\right)$, and $\Pi=\Pi^{\prime} \oplus \Pi^{\prime \prime}$. $\Pi$ is irreducible if the only subrepresentation of $\Pi$ that is non zero is $\Pi$ itself. We have the following theorem.

Theorem 4. (i) $\Pi$ is irreducible if and only if $\operatorname{Hom}(\Pi, \Pi)=\mathbf{C}$. (ii) Let $\Pi$ be a unitary representation of $\left(G_{0}, \mathfrak{g}\right)$ and let $P^{X}$ be the spectral measure of $\overline{\rho(X)}$ for $X \in \mathfrak{g}_{1}$. If $\mathcal{H}^{\prime}$ is a closed super linear subspace of $\mathcal{H}^{\prime}$, then the following statements are equivalent: $(a) \mathcal{H}^{\prime}$ is stable under $\pi_{0}$ and $\mathcal{H}^{\prime} \cap C^{\infty}\left(\pi_{0}\right)$ is stable under all $\rho(X)\left(X \in \mathfrak{g}_{1}\right)(b) \mathcal{H}^{\prime}$ is stable under $\pi_{0}$ and all the projections $P_{F}^{X}, F$ Borel and $\subset \mathbf{R}$. In particular, $\Pi$ 
is irreducible if and only if there is no nonzero proper closed super linear subspace invariant under $\pi_{0}$ and all $P_{F}^{X}\left(X \in \mathfrak{g}_{1}\right)$.

2.3. Some comments on the proofs. The most striking aspects of the above theorems are the essential self adjointness of the $\rho(X)\left(X \in \mathfrak{g}_{1}\right)$ and the fact that $C^{\infty}\left(\pi_{0}\right) \subset D(\overline{\rho(X)})$. It is clearly because of these facts that no matter what subspace $\mathcal{B}$ we start from, all the operators $\rho(X)$ extend naturally to $C^{\infty}\left(\pi_{0}\right)$ and we obtain a unitary representation in the precise sense we have defined. I shall try to explain the idea behind proving these facts.

First let us introduce some definitions. If $A$ is a closable operator and $\mathcal{D} \subset D(A)$ a dense linear subspace, we say that $\mathcal{D}$ is a core for $A$ if $A$ is the closure of its restriction to $\mathcal{D}$. Let $A$ be symmetric. We say that a vector $\psi \in D(A)$ is analytic for $A$ if $\psi \in D\left(A^{n}\right)$ for all $n$ and the series $\sum_{n} t^{n}\left\|A^{n} \psi\right\| / n$ ! converges for some $t>0$. It is a well known result that if $\mathcal{A} \subset D(A)$ contains a dense set of vectors analytic for $A$ then $A$ is essentially self adjoint and $\mathcal{A}$ is a core for $A$.

In SUSY quantum mechanics the key assumption is that the Hamiltonian $H=L^{2}, L$ being an odd symmetric operator. It is usually suggested that this assumption implies the positivity of the energy operator $H$. Actually this condition has deep consequences, not just to the positivity of the energy but to our entire theory. Let us consider the context of Theorem 2. Then, for $X \in \mathfrak{g}_{1}$, we have on $\mathcal{B}$,

$$
2 \rho(X)^{2}=\rho([X, X])=-i d \pi_{0}([X, X]) .
$$

Now

$$
U_{t}=\pi_{0}(\exp t[X, X])=e^{i t K}
$$

where $K$ is self adjoint by Stone's theorem. Under our hypotheses

$$
K=2 \rho(X)^{2}
$$

on $\mathcal{B}$. The key lemma in the proofs of the theorems stated above is the following.

Lemma. Let $H$ be a self adjoint operator and $U_{t}=e^{i t H}$. Let $\mathcal{A} \subset$ $D(H)$ be a dense linear subspace. Assume that $\mathcal{A}$ satisfies one of the two conditions: (a) $\mathcal{A}$ is invariant under the one-parameter group $\left(U_{t}\right)(b) \mathcal{A}$ contains a dense set of vectors analytic for $H$. We then have the following. 
(i) $\mathcal{A}$ is a core for $H$.

(ii) Let $L$ be a symmetric operator with $\mathcal{A} \subset D(L)$ such that $L \mathcal{A} \subset$ $D(L)$ (so that $\mathcal{A} \subset D\left(L^{2}\right)$ ) and $L^{2}$ coincides with $H$ on $\mathcal{A}$. Then $L$ is essentially self adjoint and $\mathcal{A}$ is a core for it. Moreover, $H=\bar{L}^{2}$. In particular, $H \geq 0, D(H) \subset D(\bar{L})$.

Remark. This lemma not only gives the precise conditions under which we can say that a SUSY Hamiltonian is positive, but also shows how the "odd square root" of the Hamiltonian is itself controlled by the Hamiltonian. 


\section{Super systems of imprimitivity on}

\section{purely even super homogeneous spaces}

\subsection{Special subgroups and associated super systems of imprim-} itivity. For ordinary Lie groups the central results of representations of semi direct product Lie groups follow as an application of the imprimitivity theorem which establishes a functorial equivalence between the category of unitary representations of a closed subgroup $H_{0}$ of the Lie group $G_{0}$ and systems of imprimitivity for $G_{0}$ based on $\Omega=G_{0} / H_{0}$. Actually the theory is valid in the wider category of locally compacts second countable groups but we are interested only in the case of Lie groups. Also we shall assume that $G_{0} / H_{0}$ has a $G_{0}$-invariant measure although this is not an essential restriction. A system of imprimitivity (SI) for $G_{0}$ based on $\Omega$ is noting more than a representation of the $G_{0}$-action on $\Omega$. More precisely it is a pair $(\pi, P)$ that consists of a unitary representation $\pi$ of $G_{0}$ in a Hilbert space $\mathcal{H}$ and a projection valued measure $P$ on $\Omega$ in $\mathcal{H}$ such that

$$
\pi(g) P_{E} \pi(g)^{-1}=P_{g E} \quad\left(g \in G_{0}, E \text { Borel } \subset \Omega\right) .
$$

If $\sigma$ is a unitary representation of $H_{0}$ in a Hilbert space $\mathcal{K}$, then one can associate canonically to $\sigma$ a SI $\left(\pi^{\sigma}, P^{\sigma}\right)$ for $G_{0}$ based on $\Omega$ : $\pi^{\sigma}$ is the representation of $G_{0}$ induced by $\sigma$ and $P^{\sigma}$ is the natural projection valued measure on $\Omega$ in the space of $\pi^{\sigma}$. Here we must recall that the Hilbert space $\mathcal{H}$ of $\pi^{\sigma}$ is the space of (equivalence classes of) measurable functions $f$ from $G_{0}$ to $\mathcal{K}$ such that (a) $f(x \xi)=\sigma(\xi)^{-1} f(x)$ for each $\xi \in H_{0}$ for almost all $x$ and (b) $\int|f(x)|_{\mathcal{K}}^{2} d \bar{x}<\infty$ (in view of (a) the $\mathcal{K}$-norm of $f(x)$ is really defined on $\Omega$, and $d \bar{x}$ is the invariant measure on $\Omega$.). For any Borel $E \subset \Omega$ the projection $P_{E}^{\sigma}$ is the map $f \longmapsto \chi_{E} f(f \in \mathcal{H})$ where $\chi_{E}$ is the characteristic function of $E$. The imprimitivity theorem is the statement that

$$
\sigma \longmapsto\left(\pi^{\sigma}, P^{\sigma}\right)
$$

is a functorial equivalence from the category of unitary representations of $H_{0}$ to the category of SIs based on $\Omega$. It is well known how this theorem leads in a straightforward manner to the classification of irreducible unitary representations of regular* semi direct products.

* The semi direct product $T_{0} \times{ }^{\prime} L_{0}$ is regular, $T_{0}$ being an abelian group, if the action of $L_{0}$ on the dual group $\widehat{T}_{0}$ is nice in the Borel sense, i.e., there is a Borel cross section for the orbits. 
Our intention in this section is to formulate a generalization of this result in the super setting. Exactly as in the classical theory such a generalization will lead to a classification of irreducible unitary representations of super semi direct products. To this end we work with a super Lie group $\left(G_{0}, \mathfrak{g}\right)$ and a closed super subgroup $\left(H_{0}, \mathfrak{h}\right)$. We shall assume that the subgroup is special, namely that $\mathfrak{h}_{1}=\mathfrak{g}_{1}$, or that the odd part of $\mathfrak{h}$ coincides with the odd part of $\mathfrak{g}$ ). This means that the associated homogeneous space is classical, namely $\Omega=G_{0} / H_{0}$. At this moment it is not entirely clear how to remove this restriction. However this condition is still adequate enough to provide a solution to our main problem, that of classifying the irreducible unitary representations of regular super semi direct products.

3.2. The super imprimitivity theorem. A super system of imprimitivity (SSI) based on $\Omega=G_{0} / H_{0}$ is a system $\left(\pi, \rho^{\pi}, \mathcal{H}, P\right)$ where $\left(\pi, \rho^{\pi}, \mathcal{H}\right)$ is a unitary representation of the super Lie group $\left(G_{0}, \mathfrak{g}\right),(\pi, \mathcal{H}, P)$ is a classical system of imprimitivity for $G_{0}$ based on $\Omega$, and $\rho^{\pi}$ commutes with $P$; this means that the spectral projections of the $\rho^{\pi}(X)\left(X \in \mathfrak{g}_{1}\right)$ commute with the projections $P_{E}(E$ Borel $\subset \Omega)$. The last condition, characteristic of the fact that we are dealing with special SSIs, reduces in practice to verifying the following: if $\mathcal{B}$ is a common core for all the $\rho^{\pi}(X)\left(X \in \mathfrak{g}_{1}\right)$ which is left invariant by all the $\rho(X)$ and the operators $M(u):=\int_{\Omega} u d P$ where $u \in C_{c}^{\infty}(\Omega)$, then the $\rho^{\pi}(X)$ and $M(u)$ commute on $\mathcal{B}$ in the usual sense.

We shall now associate to any unitary representation $\left(\sigma, \rho^{\sigma}, \mathcal{K}\right)$ of the super Lie group $\left(H_{0}, \mathfrak{h}\right)$, a SSI of $\left(G_{0}, \mathfrak{g}_{0}\right)$. To this end we must define $\pi$ and $\rho^{\pi}$. We shall use the flexibility provided by Theorem 3.2 and define the $\rho^{\pi}(X)$ on a suitable space $\mathcal{B}$. We define $\pi$ by

$$
\pi=\operatorname{Ind}_{H_{0}}^{G_{0}} \sigma
$$

in the Hilbert space $\mathcal{H}$ introduced earlier. Let $\mathcal{B}=C_{c}^{\infty}(\pi)$, the space of $C^{\infty}$ vectors for $\pi$ in $\mathcal{H}$ which, as functions from $G_{0}$ to $\mathcal{K}$, are smooth and have compact support mod $H_{0}$. It can be shown (as a consequence of the Dixmier-Malliavin theorem) that $\mathcal{B}$ is precisely the space of all smooth functions $f$ from $G_{0}$ to $\mathcal{K}$ such that $f(x \xi)=\sigma(\xi)^{-1} f(x)\left(x \in G_{0}, \xi \in H_{0}\right)$ and have compact support $\bmod H_{0}$. It is then easy to see that the values of any $f \in \mid b b$ lie in $C^{\infty}(\sigma)$ so that all the operators $\rho^{\sigma}(X)\left(X \in \mathfrak{h}_{1}=\mathfrak{g}_{1}\right)$ act on all the values of any $f \in \mathcal{B}$. We define

$$
\left(\rho^{\pi}(X) f\right)(x)=\rho^{\sigma}\left(x^{-1} X\right) f(x) \quad\left(f \in \mathcal{B}, x \in G_{0}\right) .
$$


Of course the projection valued measure is the standard one associated to $\pi$. We then have the following theorem.

Theorem 1. $\left(\pi, \rho^{\pi}, \mathcal{B}, P\right)$ is a $S S I$ for the super Lie group $\left.(G), \mathfrak{g}\right)$ based on $\Omega=G_{0} / H_{0}$. The assignment

$$
\left(\sigma, \rho^{\sigma}, \mathcal{K}\right) \longmapsto\left(\pi, \rho^{\pi}, P\right)
$$

is a functorial equivalence of categories from the category of unitary representations of $\left(H_{0}, \mathfrak{h}_{1}\right)$ to the category of SSIs for $\left(G_{0}, \mathfrak{g}\right)$ based on $\Omega=$ $G_{0} / H_{0}$. In particular we have a bijection from irreducible unitary representations of $\left(H_{0}, \mathfrak{h}\right)$ with irreducible SSIs for $\left(G_{0}, \mathfrak{g}\right)$ based on $G_{0} / H_{0}$. 


\section{Representations of super semi direct products}

4.1. Super semi direct products. We start with a classical semi direct product $G_{0}=T_{0} \times^{\prime} L_{0}$ where $T_{0}$ is a real finite dimensional vector space (the spacetime translation group) and $L_{0}$ a closed unimodular subgroup of $G L\left(T_{0}\right)$ acting naturally on $T$ ). We shall assume that the semi direct product is regular, namely, that for the action of $L_{0}$ on the dual $T_{0}^{*}$ there is a Borel cross section; this is equivalent to saying that all the $L_{0}$-orbits in $T_{0}^{*}$ are locally closed (Effros). Let $\mathfrak{t}_{0}=\operatorname{Lie}\left(T_{0}\right), \mathfrak{l}_{0}=\operatorname{Lie}\left(L_{0}\right), \mathfrak{g}_{0}=\operatorname{Lie}\left(G_{0}\right)$.

By a super translation group we mean a super Lie group $\left(T_{0}, \mathfrak{t}\right)$ where $\mathfrak{t}_{0}$ acts trivially on $\mathfrak{t}_{1}$. In particular this means that $\left[\mathfrak{t}_{1}, \mathfrak{t}_{1}\right] \subset \mathfrak{t}_{0}$. Suppose now that $\mathfrak{t}_{1}$ is an $L_{0}$-module and that the super commutator map $a, b \longmapsto$ $[a, b]$ is $L_{0}$ equivariant from $\mathfrak{t}_{1} \times \mathfrak{t}_{1}$ into $\mathfrak{t}_{0}$. Then $\mathfrak{g}:=\mathfrak{l}_{0} \oplus \mathfrak{t}$ is a super Lie algebra with $\mathfrak{g}_{0}=\mathfrak{l}_{0} \oplus \mathfrak{t}_{0}=\operatorname{Lie}\left(G_{0}\right), \mathfrak{g}_{1}=\mathfrak{t}_{1}$. The super Lie group $S:=$ $\left(G_{0}, \mathfrak{g}\right)$ is the super semi direct product of $L_{0}$ and the super translation group $\left(T_{0}, \mathfrak{t}\right)$. For any closed subgroup $S_{0} \subset L_{0}$ we have $H_{0}=T_{0} S_{0}$ is a closed subgroup of $G_{0}$ and $\left(H_{0}, \mathfrak{h}\right)$ is a special super Lie subgroup of $\left(G_{0}, \mathfrak{g}\right)$ where $\mathfrak{h}=\mathfrak{h}_{0} \oplus \mathfrak{t}_{1}, \mathfrak{h}_{0}$ being $\operatorname{Lie}\left(H_{0}\right)$; if $\mathfrak{s}_{0}=\operatorname{Lie}\left(S_{0}\right)$, then $\mathfrak{h}_{)}=\mathfrak{s}_{0} \oplus \mathfrak{t}_{0}$. We are interested in describing all the irreducible unitary representations of $\left(G_{0}, \mathfrak{g}\right)$. The super Poincaré groups that occur in the physical literature are special cases of the above construction where $L_{0}$ is an orthogonal group (rather its two-fold cover, the spin group) with respect to a nondegenerate quadratic form on $\mathfrak{t}_{0}$, and $\mathfrak{t}_{1}$ is chosen as a spin module for $L_{0}$. But in this section we shall discuss the general case.

The aim is to show that the irreducible unitary representations of the super semi direct product may be classified by the Frobenius-MackeyWigner method of little groups. For any $\lambda \in T_{0}^{*}$ let $L_{0}^{\lambda}$ be the stabilizer of $\lambda$ in $L_{0}$ and let

$$
\mathfrak{g}^{\lambda}=\mathfrak{t}_{0} \oplus \mathfrak{l}_{0}^{\lambda} \oplus \mathfrak{g}_{1}, \quad \mathfrak{l}_{0}^{\lambda}=\operatorname{Lie}\left(L_{0}^{\lambda}\right) .
$$

We shall refer to the super Lie group $S^{\lambda}:=\left(T_{0} L_{0}^{\lambda}, \mathfrak{g}^{\lambda}\right)$ as the super little group at $\lambda$. It is a special sub super Lie group of $S=\left(G_{0}, \mathfrak{g}\right)$. Given a unitary representation $\left(\sigma, \rho^{\sigma}\right)$ of $S^{\lambda}$, we shall say that it is $\lambda$-admissible if $\sigma(t)=e^{i \lambda(t)} I$ for all $t \in T_{0} ; \lambda$ itself is called admissible if there is an irreducible unitary representation of $S^{\lambda}$ which is $\lambda$-admissible. Let

$$
T_{0}^{+}=\left\{\lambda \in T_{0}^{*} \mid \lambda \text { admissible }\right\} .
$$


It is easy to see that $T_{0}^{+}$is $L_{0}$-invariant.

Given a unitary representation $\left(\pi, \rho^{\pi}\right)$ of the super Lie group $S$ we obtain a spectral measure on $P$ on $T^{*}$ by Fourier transformation of the restriction of $\pi$ to $T_{0}$ :

$$
\pi(t)=\int_{T^{*}} e^{i \lambda(t)} d P(\lambda) \quad(t \in T)
$$

If $E$ is any $L_{0}$-invariant Borel set, the spectrum of the unitary representation is said to be in $E$ if $P_{E}=I$.

Theorem 1. The spectrum of every irreducible unitary representation of the super Lie group $S=\left(G_{0}, \mathfrak{g}\right)$ is in some orbit in $T_{0}^{+}$. For each orbit in $T_{0}^{+}$and choice of $\lambda$ in that orbit, the assignment that takes a $\lambda$-admissible unitary representation $\gamma:=\left(\sigma, \rho^{\sigma}\right)$ of $S^{\lambda}$ into the unitary representation $U^{\gamma}$ of $\left(G_{0}, \mathfrak{g}\right)$ induced by it, is a functor which is an equivalence of categories between the category of the $\lambda$-admissible unitary representations of $S^{\lambda}$ and the category of unitary representations of $\left(G_{0}, \mathfrak{g}\right)$ with their spectra in that orbit. Varying $\lambda$ in that orbit changes the functor into an equivalent one. In particular this functor gives a bijection between the respective sets of equivalence classes of irreducible unitary representations.

Proof (Sketch). Since $T_{0}$ acts trivially on $\mathfrak{g}_{1}$ it follows that for any unitary representation $\left(\pi, \rho^{\pi}\right)$ of $S$ the operators $\pi(t)\left(t \in T_{0}\right)$ commute with $\rho^{\pi}(X)\left(X \in \mathfrak{g}_{1}\right)$ and so the spectral measure $P$ and $\rho^{\pi}$ commute with each other. If $\left(\pi, \rho^{\pi}\right)$ is irreducible and $E$ is a $L_{0}$-invariant Borel set, $P_{E}$ commutes with $\pi$ also and so $P_{E}=0$ or $I$. Thus, by regularity of the semi direct product, $P_{O}=I$ for some orbit $O$. If $\lambda \in O$ we can view $P$ as a projection valued measure on $L_{0} / L_{0}^{\lambda}$. Clearly $\left(\pi, \rho^{\pi}, P\right)$ is a SSI for $S$ based on $L_{0} / L_{0}^{\lambda}$. By the super imprimitivity theorem this is the SSI associated to a unitary representation $\left(\sigma, \rho^{\sigma}\right)$ of $S^{\lambda}$. From the classical theory one knows that $\sigma(t)=e^{i \lambda(t)} I$ for all $t \in T_{0}$. If $\left(\pi, \rho^{\pi}\right)$ is irreducible, $\left(\sigma, \rho^{\sigma}\right)$ is irreducible, and so $\lambda$ is admissible, i.e., $\lambda \in T^{+}$.

Remark. The reader should notice the sharp difference between the classical and super symmetric situations. In the classical theory, all orbits in $T_{0}^{*}$ are allowed, while in the SUSY case only those in $T_{0}^{+}$are. Actually, when everything is even, $T_{0}^{+}=T_{0}^{*}$; in fact, given any $\lambda$, the map $t x \longmapsto e^{i \lambda(t)}\left(t \in T_{0}, x \in L_{0}^{\lambda}\right)$ is well defined and gives an one dimensional irreducible unitary representation of $T_{0} L_{0}^{\lambda}$, thus showing that $\lambda \in T_{0}^{+}$. But 
when super symmetry is present, the restriction to $T_{0}^{+}$is a genuine selection rule. We shall see below that $T_{0}^{+}$may be interpreted as the condition of the positivity of energy. This is very different from the classical theory where representations of negative energy (tachyons) are theoretically possible and have to be excluded ad hoc.

Suppose $\lambda \in T_{0}^{*}$ and $\left(\sigma, \rho^{\sigma}\right)$ is a unitary representation of $S^{\lambda}$ such that $\sigma(t)=e^{i \lambda(t)} I$ for all $t \in T_{0}$. Then

$$
-i d \sigma(Z)=\lambda(Z) I \quad\left(Z \in \mathfrak{t}_{0}\right)
$$

On the other hand, if $X_{1}, X_{2} \in \mathfrak{g}_{1}$, then $\left[X_{1}, X_{2}\right] \in \mathfrak{t}_{0}$. Thus

$$
\left[\rho^{\sigma}\left(X_{1}\right), \rho^{\sigma}\left(X_{2}\right)\right]=2 \Phi_{\lambda}\left(X_{1}, X_{2}\right) I \quad\left(X_{1}, X_{2} \in \mathfrak{g}_{1}\right)
$$

where $\Phi_{\lambda}$ is the symmetric bilinear form on $\mathfrak{g}_{1} \times \mathfrak{g}_{1}$ defined by

$$
\Phi_{\lambda}\left(X_{1}, X_{2}\right)=(1 / 2) \lambda\left(\left[X_{1}, X_{2}\right]\right)
$$

If we now take $X_{1}=X_{2}=X$ we see that

$$
\rho^{\sigma}(X)^{2}=Q_{\lambda}(X) I, \quad Q_{\lambda}(X)=\Phi_{\lambda}(X, X) \quad\left(X \in \mathfrak{g}_{1}\right)
$$

on $C^{\infty}(\sigma)$. Since $\rho^{\sigma}(X)$ is essentially self adjoint on $C^{\infty}(\sigma)$ it follows that

$$
Q_{\lambda}(X) \geq 0 \quad\left(X \in \mathfrak{g}_{1}\right)
$$

This is thus a necessary condition that there should exist a unitary representation $\left(\sigma, \rho^{\sigma}\right)$ with $\sigma(t)=e^{i \lambda(t)} I$ for all $t \in T_{0}$.

Theorem 2. For $a \lambda \in T_{0}^{*}$ the following are equivalent.

(a) There is an irreducible unitary representation $\left(\sigma, \rho^{\sigma}\right)$ of $S^{\lambda}$ with $\sigma(t)=e^{i \lambda(t)} I$ for all $t \in T_{0}$.

(b) There is a unitary representation $\left(\sigma, \rho^{\sigma}\right)$ of $S^{\lambda}$ with $\sigma(t)=e^{i \lambda(t)} I$ for all $t \in T_{0}$.

(c) $Q_{\lambda}(X) \geq 0$ for all $X \in \mathfrak{g}_{1}$.

Remark. When the super semi direct product is specialized to the super Poincaré group, the condition $Q_{\lambda} \geq 0$ is the same as the positivity of the 
energy. This is the reason why we refer to this condition as the positive energy condition in the general case.

4.2. Clifford structure when $Q_{\lambda} \geq 0$. To complete the proof of Theorem 2 we must show that if $Q_{\lambda} \geq 0$ then there is an irreducible unitary representation $\left(\sigma, \rho^{\sigma}\right)$ of $S^{\lambda}$ sich that $\sigma(t)=e^{i \lambda(t)} I$ for all $t \in T_{0}$. Actually it is necessary to construct all such so that we can get a description of all irreducible unitary representations of the super semi direct product $S=\left(G_{0}, \mathfrak{g}\right)$. This depends on exploiting the Clifford structure that arises from the quadratic form $Q_{\lambda}$.

The form $Q_{\lambda}$ is invariant under $\mathrm{E}_{0}^{\lambda}$. Moreover the condition $\rho^{\sigma}(X)^{2}=$ $Q_{\lambda}(X) I$ shows that the operators $\rho^{\sigma}(X)$ are bounded self adjoint so that the situation is much nicer than for the big group. We define $\mathcal{C}_{\lambda}$ as the quotient of the tensor algebra generated by $\mathfrak{g}_{1}$ with respect to the relations $X^{2}=Q_{\lambda}(X)\left(X \in \mathfrak{g}_{1}\right)$. If $Q_{\lambda}$ is non degenerate this would be the Clifford algebra associated to the quadratic form $Q_{\lambda}$; by abuse of language we shall refer to it as the Clifford algebra associated to $Q_{\lambda}$ even if $Q_{\lambda}$ is singular.

Clearly $\rho^{\sigma}$ may now be viewed as a representation of $\mathcal{C}_{\lambda}$ in a super Hilbert space $\mathcal{K}$ such that $\rho^{\sigma}(X)$ is odd and self adjoint for all $X \in$ $\mathfrak{g}_{1}$ (SA representations). Now $L_{0}^{\lambda}$ acts on $\mathfrak{g}_{1}$ and leaves $Q_{\lambda}$ invariant. Hence its action lifts to an action $x, a \longmapsto x[a]$ on $\mathcal{C}_{\lambda}$. Then the unitary representations of $S^{\lambda}$ are pairs $(\sigma, \tau)$ where tau is an SA representation and $\sigma$ a unitary representation in the space of $\tau$ such that

$$
\tau(x[a])=\sigma(x) \tau(a) \sigma(x)^{-1} \text { for } a \in \mathcal{C}_{\lambda}, x \in L_{0}^{\lambda} .
$$

While there is nothing really difficult in the determination of the pairs $(\sigma, \tau)$, it is technically involved and I do not want to go into all the details. To simplify matters I shall assume the following condition is satisfied:

$L_{0}^{\lambda} \simeq A \times^{\prime} T, A$ simply connected and normal, $T$ a torus. (C) This condition may look artificial but in its defense let me say that it is satisfied when $S=\left(G_{0}, \mathfrak{g}\right)$ is a super Poincaré group. It is also close to the exact conditions needed for the lemma below.

Since $Q_{\lambda}$ may have a radical, say $\mathfrak{r}_{\lambda}$, we introduce

$$
\mathfrak{g}_{1 \lambda}=\mathfrak{g}_{1} / \mathfrak{r}_{\lambda}
$$


Then $Q_{\lambda}$ defines a non degenerate quadratic form on $\mathfrak{g}_{1 \lambda}$ which we denote by $Q_{\lambda}^{\sim}$. Since $L_{0}^{\lambda}$ preserves $Q_{\lambda}$ and is connected, we have a map

$$
j: L_{0}^{\lambda} \longrightarrow \mathrm{SO}\left(\mathfrak{g}_{1 \lambda}\right) .
$$

Then there are two possibilities:

(1) The map $j$ lifts to a map (this is the case when $L_{0}^{\lambda}$ is simply connected)

$$
\widehat{j}: L_{0}^{\lambda}: \longrightarrow \operatorname{Spin}\left(\mathfrak{g}_{1 \lambda}\right) .
$$

(2) $j$ does not lift as in (1) but there is a two-fold cover $H^{\lambda}$ of $L_{0}^{\lambda}$ such that $j$ lifts to a map

$$
\widehat{j}: H^{\lambda}: \longrightarrow \operatorname{Spin}\left(\mathfrak{g}_{1 \lambda}\right) .
$$

In this case the covering map

$$
H^{\lambda} \longrightarrow L_{0}^{\lambda}
$$

has kernel $\{ \pm 1\}$. We write $\xi$ for the non trivial element of this kernel. In this case we can take $H^{\lambda}=A \times^{\prime} T^{\sim}$ where $T^{\sim}$ is a torus which is a two-fold cover of $T$, and $\xi=(1, t)$. Then there is a character $\chi$ of $H^{\lambda}$ such that $\chi(\xi)=-1$.

Lemma 1. We have the following.

(a) There exist irreducible $S A$ representations of $\mathcal{C}_{\lambda}$. These are finite dimensional, unique if $\operatorname{dim}\left(\mathfrak{g}_{1 \lambda}\right)$ is odd, unique up to parity reversal if $\operatorname{dim}\left(\mathfrak{g}_{1 \lambda}\right)$ is even.

(b) Let $\tau_{\lambda}$ be an irreducible $S A$ representation of $\mathcal{C}_{\lambda}$. Then, in the presence of condition $(\mathbf{C})$ there is an even unitary representation $\kappa_{\lambda}$ of $L_{0}^{\lambda}$ in the space of $\tau_{\lambda}$ such that

$$
\tau_{\lambda}(x[a])=\kappa_{\lambda}(x) \tau_{\lambda}(a) \kappa_{\lambda}(x)^{-1} \quad\left(x \in L_{0}^{\lambda}, a \in \mathcal{C}_{\lambda}\right) .
$$

$\kappa_{\lambda}$ is unique up to multiplication by a character of $L_{0}^{\lambda}$.

Proof (Sketch). (a) is a variant of the standard theory of Clifford algebras. Let us now look at $(b)$. The spin group $\operatorname{Spin}\left(\mathfrak{g}_{1 \lambda}\right)$ is imbedded inside the even part of the Clifford algebra of $\mathfrak{g}_{1 \lambda}$ and $\tau_{\lambda}$ may be viewed as a 
representation of this Clifford algebra. Its restriction, $u$ say, to the spin group is than an even unitary representation of the spin group compatible with the action of $\mathfrak{g}_{1 \lambda}$. If now $L_{0}^{\lambda}$ is simply connected, or more generally, if $j$ lifts to a map $\widehat{j}$ as in case (1), we may take $\kappa_{\lambda}=u \circ u$. If $\widehat{j}$ exists only on a two-fold cover, $\kappa_{\lambda}^{\prime}=u \circ \widehat{j}$ defines the unitary representation we seek. It may happen that $\kappa_{\lambda}^{\prime}(\xi)=-1$ (it is \pm 1 ); in this case changing $\kappa_{\lambda}^{\prime}$ to $\chi \kappa_{\lambda}^{\prime}$ we get a unitary representation which descends to $L_{0}^{\lambda}$, which we may take as $\kappa_{\lambda}$.

Theorem 2. Let $\lambda$ be such that $Q_{\lambda} \geq 0$ and suppose that condition $(\mathbf{C})$ on $L_{0}^{\lambda}$ is satisfied. Let $\rho_{\lambda}=\left.\tau_{\lambda}\right|_{\mathfrak{g}_{1}}$, and let $\sigma_{\lambda}=e^{i \lambda} \kappa_{\lambda}$ be the unitary representation of $T_{0} L_{0}^{\lambda}$. We then have the following.

(a) $\left(\sigma_{\lambda}, \rho_{\lambda}\right)$ is an irreducible unitary representation of the little super group $S^{\lambda}$ which restricts to $e^{i \lambda}$ on $T_{0}$.

(b) The unitary representations $\left(\sigma, \rho^{\sigma}\right)$ restricting to $e^{i \lambda}$ on $T_{0}$ are precisely those of the form

$$
\sigma=\theta \otimes \kappa_{\lambda}, \quad \rho^{\sigma}=1 \otimes \rho_{\lambda}
$$

where $\theta$ is a purely even unitary representtaion of $L_{0}^{\lambda}$.

(c) The assignment

$$
\theta \longmapsto\left(\theta \otimes e^{i \lambda} \kappa_{\lambda}, 1 \otimes \rho_{\lambda}\right)
$$

is an equivalence of categories from the category of purely even unitary representations of $L_{0}^{\lambda}$ to the category of unitary representations of the little super group $S^{\lambda}$ which restrict to $e^{i \lambda}$ on $T_{0}$.

Remark 1. We have thus reached the situation where the irreducible unitary representations functorially determine the irreducible unitary representations of $S^{\lambda}$ which restrict to $e^{i \lambda}$ on $T_{0}$. Theorems 4.1.1, 4.1.2, and 4.2.2 complete the theory of unitary representations of regular super semi direct products.

Remark 2. The pair $\left(e^{i \lambda} \kappa_{\lambda}, \rho_{\lambda}\right)$ is called the fundamental representation of $S^{\lambda}$. Once it is determined, the whole structure of the irreducible unitary representations of the original super semi direct product $S$ is made transparent. The determination of $\kappa_{\lambda}$ and the verification of the condition $\mathbf{C}$ are thus the crucial ingredients that remain to be described in the super Poincaré case. 


\section{Representations of super Poincaré groups and multiplet structure of super particles}

5.1. Super Poincaré groups. Super Poincaré groups are super semi direct products that are super symmetric extensions of the Poincaré group in $D(\geq 4)$ spacetime dimensions. More precisely, in terms of the earlier notation we take

(a) $T_{0}=\mathbf{R}^{1, D-1}$, the Minkowski space of signature $(1, D-1)$ with $D \geq 4$. The Minkowski bilinear form is

$$
\langle x, y\rangle=x_{0} y_{0}-\sum_{1 \leq i \leq D-1} x_{i} y_{i} .
$$

(b) $L_{0}=\operatorname{Spin}(1, D-1)$ which is the simply connected covering group of $\mathrm{SO}(1, D-1)$. It is actually a 2 -fold cover.

(c) $\mathfrak{g}_{1}$ is a spin module for $L_{0}$, i.e., a direct sum over $\mathbf{C}$ of irreducible spin modules.

In order to have a super translation group we saw that we need a non zero symmetric $L_{0}$-equivariant bilinear form

$$
\mathfrak{g}_{1} \times \mathfrak{g}_{1} \longrightarrow \mathfrak{t}_{0}, \quad a, b \longmapsto[a, b]
$$

It can be proved that when $\mathfrak{g}_{1}$ is a spin module for $L_{0}$, such forms always exist. The classification of these forms then provides a classification of all super Poincaré groups that extend the classical Poincaré group of signature $(1, D-1)$.

The simplest situation is when $\mathfrak{g}_{1}$ is irreducible over $\mathbf{R}$. In this case the space of forms $(\mathbf{B})$ is of dimension 1. Let

$$
\Gamma^{+}=\left\{u \in \mathfrak{t}_{0} \mid u_{0}>0,\langle u, u\rangle \geq 0\right\} .
$$

Thus $\Gamma^{+}$is the closed forward light cone, origin being excluded, in $\mathfrak{t}_{0}$. It turns out that we can choose as a basis for this one dimensional space a form with the following positivity property:

$$
[X, X] \in \Gamma^{+} \quad\left(0 \neq X \in \mathfrak{g}_{1}\right) .
$$


This is remarkable because it says that for any non zero form $A$ satisfying (B), the values of the quadratic map $X \longmapsto A(X, X)(X \neq 0)$ takes values either in the forward light cone or the backeard light cone, hence by connectitivity, in one of the two entirely. It is not difficult to show that $(\mathbf{P})$ is equivalent to

$$
\langle v,[X, X]\rangle>0 \text { for all } 0 \neq X \in \mathfrak{g}_{1}, v \in\left(\Gamma^{+}\right)^{0}
$$

where the index 0 denotes interior. Using this basis element, or any other which is a positive multiple of it, we have a super Poincaré algebra (hence a super Poincaré group) in which the positivity condition $(\mathbf{P})$ is automatically satisfied. A consequence of this is the result that in any unitray representation of the super Poincaré group, the elements of $\Gamma^{+}$which can be written as sums $\sum_{j}\left[X_{j}, X_{j}\right]\left(X_{j} \in \mathfrak{g}_{1}\right)$ have nonnegative spectra. This is a form of the principle of positivity of energy.

Of course it is not necessary to assume that $\mathfrak{g}_{1}$ is an irreducible spin module. If $N$ is the number of irreducible spin modules in $\mathfrak{g}_{1}$ physicists speak of $N$-extended super symmetry when the odd commutator map

$$
\mathfrak{g}_{1} \times \mathfrak{g}_{1} \longrightarrow \mathfrak{t}_{0}, \quad X, X \longmapsto[X, X] \quad\left(0 \neq X \in \mathfrak{g}_{1}\right)
$$

takes values in $\Gamma^{+}$. The definition of super Poincaré algebra assumes this positivity. One can then classify all super Poincaré algebras. We shall not go into this classification here.

5.2. Representations of super Poincaré groups. The theory of Chapter 5 applies at once for $S=\left(G_{0}, \mathfrak{g}\right)$ a super Poincaré group. Because of the Minkowski form we can identify $\mathfrak{t}_{0}^{*}$ with $\mathfrak{t}_{0}$ itself. For $p \in \Gamma^{+}$the stabilizer $L_{0}^{p}$ is given by

$$
L_{0}^{p}= \begin{cases}\operatorname{Spin}(1, D-1) & \text { if }\langle p, p\rangle>0 \\ \mathbf{R}^{D-2} \times^{\prime} \operatorname{Spin}(D-2) & \text { if }\langle p, p\rangle=0, p_{0}>0 \\ L_{0} & \text { if } p=0\end{cases}
$$

Thus, except when $D=4, p_{0}>0,\langle p, p\rangle=0$, the stabilizer $L_{0}^{p}$ is simply connected; in the exceptional case of zero mass in 4 space time dimensions, the stabilizer is $\mathbf{R}^{2} \times^{\prime} T$ where $T$ is a circle group and so still falls within the condition $(\mathbf{C})$ described in Section 5.2.

The key lemma which shows the nature of $T^{+}$in this case is the following. 
Lemma 1. We have

$$
Q_{p} \geq 0 \Longleftrightarrow p_{0} \geq 0,\langle p, p\rangle \geq 0 \text {. }
$$

Proof (Sketch). If $v \in\left(\Gamma^{+}\right)^{0},\langle v,[X, X]\rangle>0$ for all $0 \neq X \in \mathfrak{g}_{1}$ by the structure of the super Poincaré algebra (positivity of the odd commutators). By going to the limit we see that for $p \in \Gamma^{+},\langle p,[X, X]\rangle \geq 0$ for all $X \in \mathfrak{g}_{1}$. This is just the statement that $Q_{p} \geq 0$. For the converse, suppose that $Q_{p} \geq 0$ but $\langle p, p\rangle<0$. Then $L_{0}^{p} \simeq \operatorname{Spin}(1, D-2)$. On the other hand we have a map $L_{0}^{p} \longrightarrow \mathrm{SO}\left(\mathfrak{g}_{1 p}\right)$. Since $\operatorname{Spin}(1, D-2)$ is simple and non compact it cannot have a nontrivial map into a compact group. Hence $L_{0}^{p}$ acts trivially on $\mathfrak{g}_{1 p}$. Thus the action of $L_{0}^{p}$ on $\mathfrak{g}_{1}$ contains the trivial representation of $L_{0}^{p}$. But $L_{0}$ acts as a spin module and it is not difficult to show that the action of $L_{0}^{p}$ is a sum of spin modules, and so cannot contain the trivial representation.

Theorem 2. The irreducible unitary representations of a super Poincaré group $S=\left(G_{0}, \mathfrak{g}\right)$ are parametrized by the orbits of $p$ with $p_{0} \geq 0,\langle p, p\rangle \geq$ 0 , and for such $p$, by irreducible unitary representations of the stabilizer $L_{0}^{p}$ at $p$. Let $\tau_{p}$ be an irreducible $S A$ representation of the Clifford algebra $\mathcal{C}_{p}$ and let $\kappa_{p}$ be the representation of $L_{0}^{p}$ in the space of $\tau_{p}$ defined earlier. Then, for any irreducible unitary representation $\theta$ of $L_{0}^{p}$ the pair $\left(\sigma, \rho^{\sigma}\right)$ defined by

$$
\sigma=e^{i p} \theta \otimes \kappa_{p}, \quad \rho^{\sigma}(X)=1 \otimes \tau_{p}(X) \quad\left(X \in \mathfrak{g}_{1}\right)
$$

is an irreducible unitary representation of the little super group $S^{p}=$ $\left(T_{0} L_{0}^{p}, \mathfrak{t}_{0} \oplus \mathfrak{l}_{0}^{p} \oplus \mathfrak{g}_{1}\right)$, and all irreducible unitary representations of $S^{p}$ are obtained in this manner. The unitary representation $\Theta_{p \theta}$ of the super Lie group $S$ induced by it is irreducible and all irreducible unitary representations of $S$ are obtained in this manner, the correspondence $(p, \theta) \longmapsto \Theta_{p \theta}$ being bijective up to equivalence.

Remark 1. The reader can see that in the presence of supersymmetry, only representations of positive energy come in, in contrast to the classical situation where we could not exclude the tachyonic orbits. Otherwise the reduction to the little group is exactly as before, parametrized by the irreducible unitary representations of $L_{0}^{p}$, except that it has to be tensored 
with the pair $\left(\kappa_{p}, \tau_{p},\right)$. Thus the determination of $\left(\kappa_{p}, \tau_{p}\right)$ is the basic new ingredient.

Remark 2. The correspondence with representations of the little group allows us to treat zero mass super particles with infinite spin, somehting not available in the physics literature. Also the SUSY transformations are given on the full space of super particle states, not only on those with a fixed momentum

5.3. Multiplets and fundamental multiplet. The irreducible unitary representations of the super Poincaré group are the models for super particles. If we restrict the representation of $G_{0}$, it will in general decompose into a direct sum of particles and this set is called the multiplet defined by the super particle. The operators $\rho(X)\left(X \in \mathfrak{g}_{1}\right)$ then give the transformations between particles of different spin parities. The fundamental muptiplet is the one corresponding to the trivial representation of $L_{0}^{p}$. The irreducible unitary representations into which $\kappa_{p}$ splits then define the classical particles of the fundamental multiplet.

The calculation of $\kappa_{p}$ can be carried out very explicitly. When $D=4$ and $\mathfrak{g}_{1}$ is the Majorana spinor, the multiplet with mass $m>0$ has the spins

$$
\left\{\begin{array}{l}
\{j, j, j+1 / 2, j-1 / 2(j>0) \\
\{0,0,1 / 2\}(j=0)
\end{array} .\right.
$$

For $m=0$ the mulitplet has spins (helicities)

$$
\{n / 2,(n+1) / 2\} \text {. }
$$

These results are the source for the prediction that supersymmetry forces the known elementary particles to have super partners. Whether these partners can be seen when the new LHC opens is at best speculative, since we do not know the threshold of supersymmetry breaking in the development of the early universe.

Summary. In going over from the representation theory of the Poincaré group to that of the super Poincaré group, the little group method remains valid. The additional feature is that the space of the representation of the little super group is $\mathcal{K}_{\text {classical }} \otimes \mathcal{K}_{\text {Clifford }}$. The second factor carries the action of the odd operators of the little super group, and the even representation of the little super group is $\theta \otimes \kappa_{p}$. Thus the states of the 
little group are the classical states tensored with the Clifford states; on the latter both the odd operators and the classical part of the little super group act.

This theory allows a discussion of super particles of mass 0 with infinite spin, which is new. The SUSY transformations of particle states are globally given, which is also new; the usual formulas are restricted to states of fixed momentum

\section{References}

\section{Basics}

1. Varadarajan, V. S., Supersymmetry for Mathematicians: An Introduction, AMS-Courant Institute Lecture Notes, Providence, R.I., 2004.

2. Deligne, P., and Morgan, J., Notes on supersymmetry (following Joseph. Bernstein), Quantum fields and strings: a course for mathematicians, vol. I, 41-97, American Mathematical Society, Providence, R.I.,1999.

3. Kostant, B., Graded manifolds, graded :ie theory, and prequantization in Differential geometrical methods in mathematical physics, Proc. Symp. UNiv. Bonn, Bonn, 1975, Lecture Notes in Mathematics, Springer, Berlin, 1977.

4. Leites, D., Introduction to the theory of super manifolds, Usp. Mat. Nauk. 35(1), 3-57, 1980, Russ. Math. Surveys, 35(1), 1-64, 1980.

5. Manin, Yu. I., Gauge field theory and complex geometry, Grundlehren der Mathematischen Wissenschaften, 289, Springer, Berlin, 1988. 
6. Freed, D. S., Five lectures on supersymmetry, Amer. Math. Soc., R. I., 1999.

7. Carmeli, C., Super Lie groups,. Structure and representations, Thesis, University of Genoa, Preprint, 2006.

8. Fioresi, R., and Caston, L., Super geometry, Preprint, 2006.

\section{Physics, Representations}

9. Ferrara, S., ed., Supersymmetry, vols. 1, 2. World Scientific, Singapore, 1987.

10. Dobrev, V. K., and Petkova, V. B., All positive energy unitary irreducible representations of extended conformal super symmetry, Phys. Lett. B 162(1-3), 127-132 (1985); All positive energy unitary irreducible representations of the extended conformal super algebra. In Conformal groups and related symmetries: physical results and mathematical background, Clausthal-Zellerfeld, 1985, Vol 261, Lecture Notes in Physics, Springer, Berlin, 1986, 300-308; Group theoretical approach to extended conformal super symmetry: function space realization and invariant differential operators, Fortschr. Phys. 35 (7), 537-572 (1987).

11. Carmeli, C., Cassinelli, G., Toigo, A., and Varadarajan, V. S., Unitary representations of super Lie groups and applications to the classification and multiplet structure of super particles, Comm. Math. Phys., 263(2006) 217-258.

12. Jakobsen, H. P., The full set of unitarizable highest weight modules of basic classical Lie super algebras, Memoirs of the Amer. Math. Soc., 532 (1994), Providence, R. I.

13. Witten, E., Physics and geometry, Proceedings of the Berkeley ICM, 1987, p. 267. 